\title{
Prognosis and sleep disordered breathing in heart failure
}

\author{
I Wilcox, S G McNamara, T Wessendorf, G N Willson, A J Piper, C E Sullivan
}

Abnormal breathing in heart failure as originally described by Cheyne ${ }^{1}$ and subsequently by Stokes ${ }^{2}$ was observed in apparently awake patients as an agonal breathing pattern.

"... The only peculiarity in the last period of his illness was in the state of the respiration. For several days his breathing was irregular, then it would become perceptible, though very low, then by degrees it became heaving and quick, and then it would cease again: this revolution in the state of his breathing occupied about a minute, during which there were about 30 acts of respiration (Cheyne, 1818) ..."

The patient described in this report was an obese, elderly, alcoholic who had suffered a substantial, and ultimately, fatal stroke. He probably had obstructive sleep apnoea prior to the stroke and developed central apnoea subsequently. In the literature subsequent to these reports periodic respiration associated with central apnoeas was believed to be a terminal consequence of end stage heart failure..$^{3-5}$

More recently it has been recognised that central apnoeas occur commonly in heart failure, especially during sleep, being reported in $40-50 \%$ of patients, predominantly men, with stable, medically treated congestive heart failure. ${ }^{6}$ This sleep breathing abnormality leads to sleep fragmentation, alterations in sleep architecture-with relative increases in stage 1 and 2 sleep and reduction in REM sleep - and a clinical sleep disorder with symptoms of tiredness and sleepiness in some patients. ${ }^{7}$ The sleep disorder in central sleep apnoea is a consequence of the development of congestive heart failure. Although the pathophysiology is not completely understood, hyperventilation, ${ }^{8}$ increased chemoreceptor drive, ${ }^{9}$ and increased circulation time ${ }^{41011}$ are all believed to be important factors promoting this sleep disorder.

Identification of obstructive sleep apnoea (OSA) in patients with primarily left ventricular (LV) dysfunction and clinical heart failure is a more recent observation. Pulmonary hypertension and consequent right heart failure (cor pulmonale) is well recognised to be part of the combination of obesity, hypoventilation, and severe obstructive sleep apnoea. Malone et $a l^{12}$ reported a group of patients with a clinical presentation with dilated cardiomyopathy and obstructive sleep apnoea in whom treatment with nasal continuous positive airway pressure (CPAP) was associated with improvements in symptoms of dyspnoea and left ventricular ejection fraction. These, and subsequent observations, have suggested that OSA and heart failure is an adverse combination in which OSA causes or exacerbates left ventricular dysfunction.
The disease process responsible for congestive heart failure has been reported to vary, with valvular and hypertensive heart disease being more common in earlier reports. Currently, dilated cardiomyopathy and coronary heart disease account, in approximately equal proportions, for most of the patients with end stage heart failure awaiting cardiac transplantation. ${ }^{13}$ The survival of patients who present with congestive heart failure is poor, with reported mortality rates of $50 \%$ at 12 months in those with severe symptoms and $50 \%$ at 3-4 years in those with less severe heart failure, ${ }^{14}$ a prognosis which is similar to that of many patients with malignancy.

Prognostic factors in patients with end stage congestive heart failure ${ }^{13}$ include haemodynamic (cardiac output, pulmonary vascular resistance), neurohumoral (increased sympathetic nerve activity), electrophysiological (spontaneous ventricular tachycardia), and treatment factors (converting enzyme inhibitors, $\beta$ blockers). Sleep disordered breathing has not been regarded as a prognostic factor in heart failure previously, although there is increasing evidence to suggest that it may be (table 1 ).

\section{Heart failure and central sleep apnoea}

Central sleep apnoea and Cheyne-Stokes respiration (CSR) represent a spectrum of severity from intermittent periodic respiration without apnoeas to cycles of hyperventilation and apnoea during sleep. The severity of the breathing disturbance is reduced by REM sleep and suppressed by the onset of wakefulness. Post-apnoeic hyperventilation with arousal early or at the peak of hyperventilation is recognised clinically as paroxysmal nocturnal dyspnoea as was originally described by Harrison. ${ }^{3}$

The clinical characteristics of patients with central sleep apnoea/CSR reported in the literature have generally been older, not obese, with at least moderately severe LV dysfunction (LV ejection fraction $<30 \%$ ) and with relatively few patients with dilated cardiomyopathy. In early reports hypertensive and valvular disease was common, ${ }^{411}$ but this has been less so in more recent reports (table 2). Coronary heart disease, approximately equal to dilated cardiomyopathy as a cause of end stage heart failure in patients on transplant waiting lists, ${ }^{13}$ is the underlying

Table 1 Risk indicators in patients awaiting cardiac transplantation

- Haemodynamic

- Neurohumoral

- Electrophysiological

- Treatment

- Sleep apnoea?

Modified from Stevenson. ${ }^{13}$
Alfred Hospital, Missend

Road, Camperdown, NSW

2050, Australia. 
Table 2 Cheyne-Stokes respiration and heart failure: patient characteristics

\begin{tabular}{llll}
\hline Author & Sex & Aetiology & LVEF \\
\hline Prior $(1951)^{4}$ & $5 \mathrm{M}$ & HT 3, DCM, LVH & CXR \\
Lange and Hecht $(1962)^{11}$ & $8 \mathrm{M}$, 1F & CAD 5, V 2, HT 2 & CXR \\
Hanly et al $(1989)^{7}$ & $10 \mathrm{M}$ & CAD 9 & $22(5) \%$ \\
Takasaki et al $(1989)$ & $5 \mathrm{M}$ & CAD 4, DCM 1 & $31(8) \%$ \\
Javaheri et al $(1995)^{6}$ & $19 \mathrm{M}$ & CAD 13, DCM 6 & $22(9) \%$ \\
Naughton et al $(1995)^{8}$ & $25 \mathrm{M}$ & CAD 21, DCM 3 & $20(3) \%$
\end{tabular}

$\mathrm{LVEF}=\mathrm{LV}$ ejection fraction, $\mathrm{CXR}=$ radiological evidence of heart failure, $\mathrm{HT}=$ hypertension, $\mathrm{LVH}=$ idiopathic LV hypertrophy, CAD = coronary artery disease, DCM = dilated cardiomyopathy, $\mathrm{V}=$ valvular disease.

cardiac pathology in most patients with central sleep apnoea. Almost none of the patients with central apnoea are women, which may reflect selection bias but has been consistently reported by all investigators and may reflect sex differences in coronary heart disease or other factors such as differences in ventilatory control.

Central sleep apnoea appears to occur commonly in heart failure, having been reported in $40-60 \%$ of patients with stable congestive heart failure.$^{56}$ In a prospective Veterans Administration study Javaheri et $a l^{6}$ evaluated the prevalence of sleep disordered breathing in a cohort of 42 patients with optimally treated stable congestive heart failure and divided the patients into those with (group II, $\mathrm{n}=19,42 \%$ ) and those without (group I, $\mathrm{n}=23,55 \%$ ) an apnoea/ hypopnoea index (AHI) of $>20 / \mathrm{h}$. The patients in group II had an AHI of 44 (13)/h. Sleep studies were performed carefully including sham and clinical studies. Variables measured included Holter recordings for ventricular arrhythmias as well as LV ejection fraction. The occurrence of sleep apnoea was compared with clinical, Holter, and LV ejection fraction and other "pathophysiologically important" variables using multivariable analysis. There were no differences in age and obesity (body mass index, $\mathrm{BMI}$ ) between patients with and without repetitive, predominantly central sleep apnoea.

Obstructive events were reported in a minority of group I patients and were positively correlated with BMI. The presence of central apnoeas (AHI $>20 / \mathrm{h}$ ) was correlated inversely with LV ejection fraction. The mean age of the patients was relatively elderly, not surprisingly in a Veterans Administration study, and was similar in both groups. Importantly, but difficult to examine systematically in a study of this size, ventricular arrhythmias were more frequent and complex during $24 \mathrm{~h}$ Holter ECG recordings. Ventricular tachycardia-known to be an important adverse prognostic factor in heart failure - was an order of magnitude more frequent in patients with central sleep apnoea. The increased propensity to ventricular tachycardia may have been influenced by the relative increase in sympathetic nervous system activity associated with repetitive cycles of apnoeas, hyperpnoea, and arousals from sleep. ${ }^{15}$

In considering prognosis, end points other than death such as quality of life, which is known to be affected by sleep fragmentation of any cause, and morbidity such as readmission to hospital or referral for transplantation need to be considered along with cardiac deaths. In addition, congestive heart failure is associated with an increased risk of non-cardiac end points such as stroke.

The first systematic study of prognosis in heart failure and central sleep apnoea was reported by Findley and coworkers in $1985 .{ }^{5}$ These investigators studied 15 men with stable congestive heart failure (due to coronary disease in 13 and valvular heart disease in 2) in two centres (Denver, Colorado and San Diego, California). Six $(40 \%)$ had more than five apnoeas per hour of sleep (mean 28/h). During six months of follow up all six men with central sleep apnoea died compared with only $33 \%$ in the group without central sleep apnoea. Patients with central sleep apnoea were not matched for important clinical variables such as age (mean 68 years versus 58 years, $\mathrm{p}=\mathrm{NS}$ ) and ejection fraction (28 (3)\% versus 34 (9)\%, $\mathrm{p}=\mathrm{NS})$. These differences in baseline variables were not statistically significant but it appears likely that the study was underpowered to demonstrate possible differences. The trends towards increasing age and worse ventricular function would predict a worse prognosis, but not to the degree observed in only six months of follow up.

Hanly et $a l^{16}$ prospectively evaluated 16 men with severe symptomatic congestive heart failure (NYHA class III or IV) due to coronary heart disease, with $(n=9)$ or without $(n=7)$ CSR, and followed them over a period of 3.14.5 years using telephone follow up (fig 1). Patients with CSR did not differ with respect to circulation time (19 (4) s versus 16 (7) s) and LV ejection fraction (22 (5)\% versus 24 (5)\%, CSR versus no CSR respectively). Five of the patients $(55 \%)$ with CSR died and two were referred for cardiac transplantation (total adverse events $78 \%$ ) compared with only one of seven men $(14 \%)$ without CSR $(p<0.05$ for pooled adverse events). Regression analysis showed that the risk of cardiac death or transplantation increased with central sleep apnoea, AHI, arousal index and the amount of stage 1 and 2 sleep, and inversely with the total sleep time. The number of patients included in this study was not sufficient to allow multivariable analysis.

Wilcox $e t a l^{17}$ studied 26 consecutive patients who presented with a clinical sleep disorder, impaired LV function, and central sleep apnoea ( $>75 \%$ of respiratory events were central with apnoea/hypopnoea index (AHI) of 44 (14) events/h and minimum $\mathrm{SaO}_{2}$ of 79 (8)\%). All patients were men with a mean (SD) age of 65 (9) years, and most were not obese (BMI 27 (4) $\mathrm{kg} / \mathrm{m}^{2}$ ). Most patients had significant dyspnoea

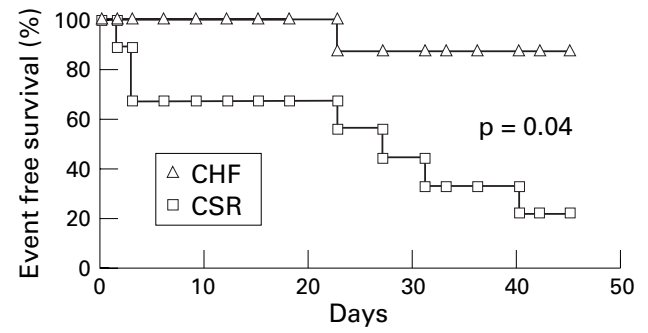

Figure 1 Event free survival (days) in patients with heart failure with (CSR) and without Cheyne-Stokes respiration $(\mathrm{CHF})$. Modified from reference 16. 
Table 3 Central sleep apnoea and heart failure

Summary

- Apparent excess of men

- Common, occurs in 40-50\% of stable patients with CHF

- Severe LV dysfunction (mean LVEF 20\%)

- Cause of heart failure is predominantly coronary artery disease

- Sleep breathing disorder is a consequence of the heart disease.

- Treatment may include modalities other than CPAP including oxygen, theophylline, nasal ventilation

$\mathrm{CHF}=$ congestive heart failure $\mathrm{LVEF}=$ left ventricular ejection fraction; $\mathrm{CPAP}=$ continuous positive airway pressure.

(mean NYHA class $3.2(0.1)$ ) and severe LV dysfunction (LV ejection fraction $22(9) \%$ ) due to coronary artery disease (14 patients, 54\%), dilated cardiomyopathy (eight patients, $31 \%$ ), or valvular heart disease (four patients, $15 \%$ ). All patients were receiving standard medical therapy with diuretics, digoxin, and converting enzyme inhibitors. Treatment with nasal CPAP and/or low flow oxygen was administered in 13 cases $(50 \%)$.

During follow up of 22 (14) months eight of 26 men $(31 \%)$ died from cardiac causes and none died from a non-cardiac cause. Nine patients $(35 \%)$ were readmitted to hospital with worsening heart failure. These men clearly had an adverse prognosis although it was not necessarily worse than patients without central sleep apnoea and heart disease of equivalent severity. The prognosis of men in our study was similar to those reported by Hanly et $a l^{16}$ although they pooled referral for cardiac transplantation with death as an end point.

End points other than death include exacerbations of heart failure requiring readmission to hospital and possibly stroke, which are common factors adversely affecting morbidity in patients with heart failure. In our study $35 \%$ of the patients were admitted to hospital with worsening heart failure, which indicates a severe exacerbation in heart failure given that most of such episodes are managed as outpatients in current practice.

In a study of 36 patients with congestive heart failure and a mean central apnoea/hypopnoea index of $22 / \mathrm{h}$, Andreas et $a l^{18}$ reported that the presence of central sleep apnoea during sleep was not an adverse prognostic factor whereas daytime periodic respiration was. However, patients with periodic respiration while awake had both a more severe form of this sleep breathing disorder and poorer left ventricular function. Since LV dysfunction is one of the most powerful adverse prognostic factors in heart failure, any independent effect of severity of central apnoea would be hard to assess in a relatively small study with few end points.

Central sleep apnoea or Cheyne-Stokes respiration is a consequence of the development of congestive heart failure in patients who may or may not have had prior obstructive sleep apnoea (table 3). The spectrum of the disorder varies in severity from periodic respiration during sleep without frank apnoeas to repetitive cycles of apnoea and hyperventilation during wakefulness. Patients with heart failure who also have central sleep apnoea have a seriously adverse prognosis which is not as poor as early reports suggested, but may be worse than patients with- out central apnoea when other prognostic factors are adjusted for. Effective treatment of this sleep disorder include oxygen, nasal CPAP, theophylline, and nasal ventilation. These treatments can reduce sleep fragmentation and have the potential to improve both quality of life and survival in patients with heart failure and central sleep apnoea.

Heart failure and obstructive sleep apnoea The prognosis of patients with obstructive sleep apnoea and heart failure has been less extensively studied than for central sleep apnoea. However, surrogate end points such as improvement in functional class and LV ejection fraction have been reported in a series of publications by Bradley and coworkers in Toronto. Treatment of sleep apnoea was associated with improved NYHA functional class and LV ejection fraction within eight weeks of treatment with nCPAP. These effects have been shown to be reversed by treatment withdrawal and restored with continuing therapy. ${ }^{12}$

Obstructive sleep apnoea in patients without heart failure is associated with cyclical increases in sympathetic nerve activity which accompany apnoeas, ${ }^{19}$ persist during wakefulness, and are reduced by CPAP therapy. ${ }^{20}$ Similarly, blood pressure variability and probably levels are reduced by effective treatment of obstructive sleep apnoea. ${ }^{21-23}$ Increased afterload is poorly tolerated by the failing heart and afterload reducing agents such as converting enzyme inhibitors improve symptoms and survival in congestive heart failure. As NYHA class and ejection fraction are two important independent prognostic factors in heart failure, it is reasonable to postulate that these changes could translate into a survival benefit. However, this potential prognostic benefit cannot be assumed as previous studies with drugs such as inotropic agents, which improve acute haemodynamics, have consistently led to impaired survival during chronic use. ${ }^{24}$

We studied 25 consecutive patients who presented with a clinical sleep disorder, impaired $\mathrm{LV}$ function and obstructive sleep apnoea $(>75 \%$ of respiratory events were obstructive). ${ }^{17}$ These patients were studied consecutively and concurrently with patients with predominantly central sleep apnoea. They included one woman, were significantly younger (54 (7) years) than men with central sleep apnoea $(65$ (9) years, $\mathrm{p}<0.001)$, but had sleep apnoea of similar severity (AHI 40 (25) events/h and minimum $\left.\mathrm{SaO}_{2} 73(13) \%\right)$ to patients with central sleep apnoea. Patients with obstructive sleep apnoea were obese (BMI 34 (6) $\left.\mathrm{kg} / \mathrm{m}^{2}\right)$, slightly less symptomatic (NYHA class $2.6(0.9)$ ), and had less severe LV systolic dysfunction (LV ejection fraction 29 (9)\%) than patients with central sleep apnoea of similar severity (LV ejection fraction 22 $(9) \%, p=0.05)$. There appeared to be a trend towards a greater proportion of patients with dilated cardiomyopathy (16/25, patients, $64 \%)$ and fewer with coronary artery disease $(8 / 25$, $32 \%)$, only one patient $(5 \%)$ had valvular heart disease. 
Table 4 Obstructive sleep apnoea and heart failure

Summary

- Apparent excess of men

- Broader range of LV systolic dysfunction

- Possibly worse diastolic function

- Appears to have better prognosis than CSA/CSR

- Cause of heart failure is predominantly dilated

cardiomyopathy

- Heart disease presumed to be caused or exacerbated by OSA

- Nasal CPAP is treatment of choice

CSA = central sleep apnoea; CSR = Cheyne-Stokes respiration $\mathrm{OSA}=$ obstructive sleep apnoea $\mathrm{CPAP}=$ continuous positive airways pressure.

Patients received standard drug treatment for heart failure and treatment with nasal CPAP was administered in 18 of the 25 patients $(72 \%)$. CPAP was either not tolerated or not obtained for the remaining untreated patients. During follow up of 22 (14) months none died and nine $(35 \%)$ were readmitted to hospital with worsening heart failure. The combination of obstructive sleep apnoea and congestive heart failure in this study identified patients with a markedly different outcome in terms of mortality, and probably morbidity, despite similarly severe sleep apnoea to that of a simultaneously studied cohort of men with central sleep apnoea. The findings in the present study are only applicable to men because only one woman was included.

Obstructive sleep apnoea in heart failure (table 4) identifies patients in whom sleep apnoea either causes or contributes to impairment of LV function. These patients are more likely to have dilated cardiomyopathy as their underlying pathology, tend to have less severe LV dysfunction, and a better prognosis with and possibly without treatment. Treatment of OSA with nasal CPAP improves symptoms, morbidity, left ventricular function, and may improve survival.

\section{Conclusion}

Sleep apnoea syndromes associated with congestive heart failure may be either obstructive or central sleep apnoea. We suggest that these are two fundamentally different clinical syndromes of sleep apnoea and heart failure with important differences in pathophysiology, treatment and, most importantly, prognosis. Identification of the presence and type of sleep disordered breathing is potentially a major new prognostic factor in patients with congestive heart failure.
Supported by a project grant from the National Health and Medical Research Council of Australia.

1 Cheyne J. A case of apoplexy, in which the fleshy part of the heart was converted into fat. Dublin Hospital Reports $818 ; 2: 216-23$

2 Stokes W. The diseases of the heart and aorta. Dublin: Hodges and Smith, 1854: 302-37.

3 Harrison TR, King CE, Calhoun JA, et al. Congestive heart failure: Cheyne-Stokes respiration as the cause of paroxysmal dyspnoea at the onset of sleep. Arch Intern Med 1934; 53:891-910.

4 Prior WW. Cheyne-Stokes respiration in patients with cardiac enlargement and prolonged circulation time. Circulation 1951;4:223-38.

5 Findley LJ, Zwillich CW, Ancoli-Israel S, et al. CheyneStokes breathing during sleep in patients with left ventricular heart failure. South Med f 1985;78:11-15.

6 Javaheri S, Parker TJ, Wexler L, et al. Occult sleep disordered breathing in stable congestive heart failure. Ann Intern Med 1995;122:487-92.

7 Hanly PJ, Millar TW, Steljes DG, et al. Respiration and abnormal sleep in patients with congestive heart failure. Chest 1989; 96:480-8

8 Naughton M, Benard D, Tam A, et al. Role of hyperventilation in the pathogenesis of central sleep apneas in patients with congestive heart failure. Am Rev Respir Dis 1993;148: $330-8$.

9 Wilcox I, Grunstein RR, Berthon-Jones M, et al. Role of central chemosensitivity in central sleep apnoea associated with heart failure. Sleep 1993;16:S37-9

10 Guyton AC, Crowell JH, Moore JW. Basic oscillating mechanism of Cheyne-Stokes breathing. Am F Physiol 1956;187: 385-98.

11 Lange RL, Hecht $\mathrm{H}$. The mechanism of Cheyne-Stokes respiration. F Clin Invest 1962;41:42-52.

12 Malone S, Lui P, Holloway R, et al. Obstructive sleep apnoea in patients with dilated cardiomyopathy: effects of continuous positive airway pressure. Lancet 1991;338:1480-4.

13 Stevenson LW. Selection and management of patients for cardiac transplantation. Curr Opin Cardiol 1993;8:411-8. 14 Cowie MR, Mosterd A, Wood DA, et al. The epidemiology Cowie MR, Mosterd A, Wood DA, et al. The

15 Naughton MT, Bernard DC, Liu PP, et al. Effects of nasal CPAP on sympathetic activity in patients with heart failure and central sleep apnea. Am $\mathcal{F}$ Respir Crit Care Med 1995;152:473-9.

16 Hanly PJ, Zuberi-Khokhar NS. Increased mortality associated with Cheyne-Stokes respiration in patients with congestive heart failure. Am $\mathcal{f}$ Respir Crit Care Med 1996;153:272-6.

17 Wilcox I, McNamara SG, Willson GN, et al. Is sleep apnea a new prognostic marker in heart failure? Circulation 1995; 92:I-274.

18 Andreas S, Hadenah G, Moller C, et al. Cheyne-Stokes respiration and prognosis in congestive heart failure. $\mathrm{Am} \mathcal{F}$ Cardiol 1996;78:1260-4

19 Hedner J, Ejnell H, Sellgren J, et al. Is high and fluctuating muscle nerve sympathetic activity in the sleep apnea muscle nerve sympathetic activity in the sleep apnea syndrome of pathogenetic importance for the

20 Carlson J, Hedner JA, Elam M, et al. Augmented Carlson J, Hedner JA, Elam M, et al. Augmented sympathetic activity in awake patients
sleep apnea. Chest 1993;103:1763-8.

21 Jennum P, Wildsciodtz G, Christensen NJ, et al. Blood pressure, catecholamines and pancreatic polypeptide in obstructive sleep apnea with and without nasal continuous positive airway pressure (nCPAP) treatment. $\mathrm{Am} f$ Hypertens 1989;2:847-52.

22 Mayer J, Becker H, Brandenburg U, et al. Blood pressure and sleep apnea: results of long-term nasal continuous positive airways pressure therapy. Cardiology 1991;79:8492 .

23 Wilcox I, Grunstein RR, Hedner JA, et al. Effect of nasal continuous positive airway pressure during sleep on 24-hour blood pressure in obstructive sleep apnea. Sleep 1993;16:539-45.

24 Chatterjee K. Heart failure therapy in evolution. Circulation 1996;94:2689-93. 